\title{
Isolated Handwritten Eastern Arabic Numerals Recognition Using Support Vectors Machines
}

\author{
B. El Kessab*, C. Daoui, B. Bouikhalene, R. Salouan \\ Laboratory of Information Processing and Decision Support, Faculty of Science and Technology, \\ BP 523, BeniMellal, Morocco \\ *Corresponding author, email: bade10@hotmail.fr
}

\begin{abstract}
In this paper, we present a comparison between the different variations of virtual retina (grid size) in features extraction with the support vectors machines classifier for isolated handwritten Eastern Arabic numerals recognition. For this purpose we have used for pre-processing each numeral image the median filter, the thresholding, normalization and the centering techniques. Furthermore, the experements results that we have obtained demonstrate really that the most powerful method is that virtual retina size equal 20x20. This work has achieved approximately $85 \%$ of success rate for Eastern Arabic numerals database identification.
\end{abstract}

Keywords: isolated handwritten eastern arabic numerals, median filter, thresholding, centering, normalization, retinal coding method, and the support vectors machines

Copyright $@ 2015$ Institute of Advanced Engineering and Science. All rights reserved.

\section{1, Introduction}

Optical Character Recognition (OCR) is considered recently as a very dynamic field given that its applicability in many different domains such as postal sorting, bank cheque processing and automatic data entry, etc. Moreover, the OCR can be applied on both cases printed or handwritten. In fact recognition for handwritten case is more complex than that printed due to varying writing styles from person to another even so just for one given pferson which will make this kind of recognition very difficult which requires for resolving this problem to use several effecient techniques in each of the three principal phases forming a certain system of recognition which are firstly the pre-processing then secondly the features extraction then finally learning and classification or quite simply recognition. In this framwork, several studies has been done for recognition of isolated handwritten Arabic or Latin character or numerals by using in the features extraction phase the retinal coding method in one hand or in the learningclassification phase the support vectors machines [6-8] on the other hand. Hence, concerning this approach, we are interested to isolated handwritten Eastern Arabic numerals recognition.

Therefore, in this sense and in order to achieve this task we have pre-processed each numeral image by the median filter, the thresholding, the centering and the normaization techniques while we extracted the features of each numeral by the retinal coding, about the recognition of each unknown numeral we have used the support vectors machines. In fact, our targeted purpose is being able to compare between the precision of the different variations of grid sizes in features extraction with the support vectors machines classifier on the other side for isolated handwritten Eastern Arabic numerals recognition. Anyway, this paper is organized in the following manner. First, in section 1 the proposed recognition system is schematized, Section 2 describes techniques for image pre-processing. Section 3 introduces the retinal coding. In Section 4, the support vectors machines classifier is presented. Section 5 shows the experimental results. Finally, the study is ended by a conclusion.

\section{Recognition System} figure:

The recognition system that we have opted in this study ispresented in the following 


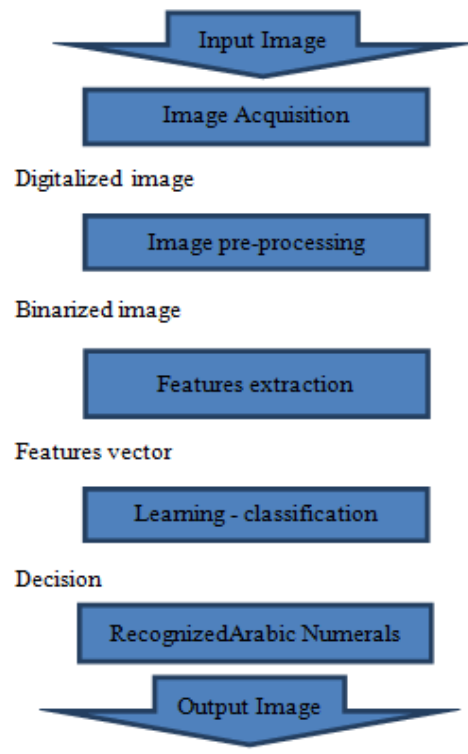

Figure 1. The proposed recognition system

\section{Pre-Processing}

The first phase in each OCR system the pre-processing whose the goal is to remove each needless pixel including noise and redundant information in order to render in a best quality the numeral image so that it can be used in an efficient manner in the following phase which is the features extraction. Of this fact, to achieve this task, we have pre-processed in this research the images by the following techniques:

1) The median filter applied for performing a filtration of image.

2) The thresholding used to render each image contains only the black and white colors according a pre-selected threshold.

3) The centering exploited for localizing the numeral justly in center of its image. The normalization with standard size of each numeral image.

\section{Features Extraction}

Features extraction play enormously a very important role in each OCR system, especially for handwritten optical character recognition, in fact the precision of an certain system recognition depends heavily to features extraction operation in reason of if an great discrmination between characters is truly realized its recognition will be at that time very correct. More precisely, feature extraction methods can be divided into two principal categories: structural [10-17] and statistical [1-5] features The first category is based on local structure of numeral image while the second is interested to statistical information's localized in character image by way of example within this context there are the moments of images especially those invariants.

In this framework, we have chosen a structural method which is Retinal coding method.

\subsection{Retinal Coding}

The process of retinal coding that we have used is explained as follow:

Each image is a black containing a numeral writting in white color and has firstly an size equal to $30 \times 30$ pixels. First of all, given a virtual grid or retina having a size equal to $2 N / 3 \times$ $2 \mathrm{~N} / 3$ pixels while this last of each numeral image is equal to $\mathrm{NxN}$ pixels, therefore in order to applied this method as it should the image must be resized to2 $\mathrm{N} \times 2 \mathrm{~N}$ pixels, afterwards the retina is placed on the first zone of image the on second zone and so on until the last zone while at starting from the top located to the left of the image in each putting in zone of the retina the number of white pixels is calculated which will allow thereafter ultimately to convert the image to a vector. 
Morever, in order to well fix the ideas the schema below illustrates this mechanism (we take $\mathrm{N}=12)$ :

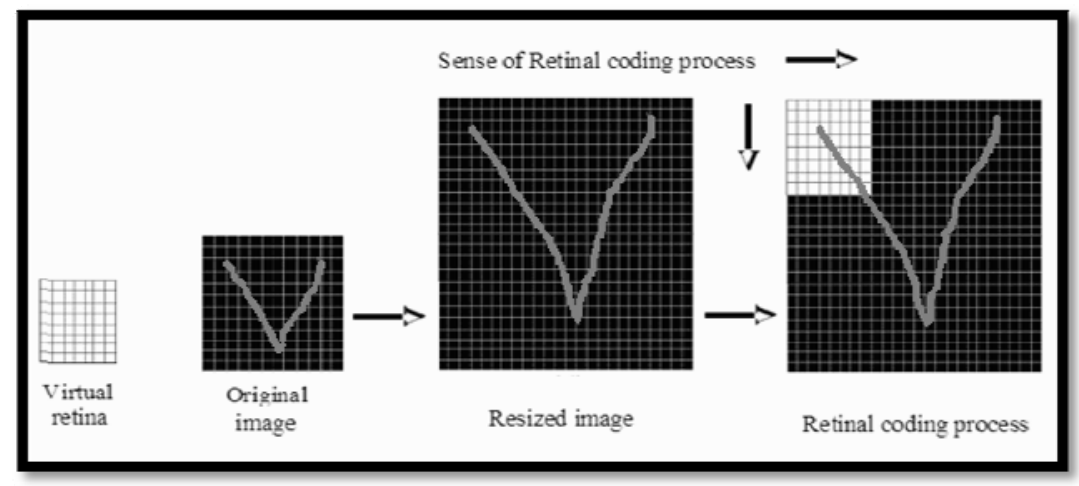

Figure 2. The process of retinal coding method

\section{Recognition}

An SVM [6-8] is considered as an statistical and supervised method it is basically defined for two-class problem separation, and it finds an optimal hyperplane which can maximize the margin between the nearest examples of both classes, named support vectors (SVs).

First of all, given a training database of $M$ data: $X_{i}, i=1,2 \ldots . . M$.

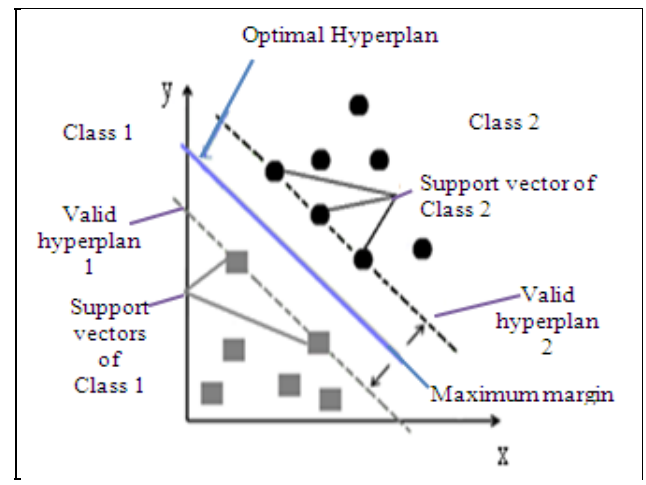

Figure 3. The determination of optimal hyperplane, vectors supports, maximum Marge and valid hyperplanes

The linear SVM classifier is then defined as:

$$
\begin{aligned}
& f(X, w, b): x \longrightarrow y \\
& f(X)=w X+b
\end{aligned}
$$

Where $\mathrm{w}$ and $\mathrm{b}$ are the parameters of the classifier $\mathrm{y}$ is the label.

The linear SVM can be extended to a non-linear classifier by replacing the inner product between the input vectors xand the SVMs, through a kernel function K defined as: 
Table 1. Examples of different kernel functions used in SVM

\begin{tabular}{ll} 
Kernel linear & $x y$ \\
Kernel polynomial of degree $\mathrm{n}$ & $(\text { axy }+b)^{n}$ \\
Gaussian radial basis function (GRBF) of a standard deviationo: & $e^{-\frac{\|x-y\|^{2}}{2 \sigma^{2}}}$ \\
\hline
\end{tabular}

The method described above is designed for a problem of two classes only, many studies treat a generalization of theSVM to a multi-classification [8] among these studies we cite the two strategies frequently used: the first approach isbased to use $\mathrm{N}$ decision functions (one against all) allowing to make a discrimination of a class contains a one vector labeled by the value 1 againstall other vectors existed in a other class opposite having a label equal to -1 . Therefore the decision rule used in this case is usually the maximum such that wewill assign an unknown vector $\mathrm{X}$ into a class associated with an output of SVM is the largest.

$$
\text { Classe }(X)=\arg \max _{i=1,2, \ldots, n} f_{i}(x)
$$

\section{Experiments and Results}

First of all, we present an example of some Eastern Arabic handwritten numerals that we have used in our study:

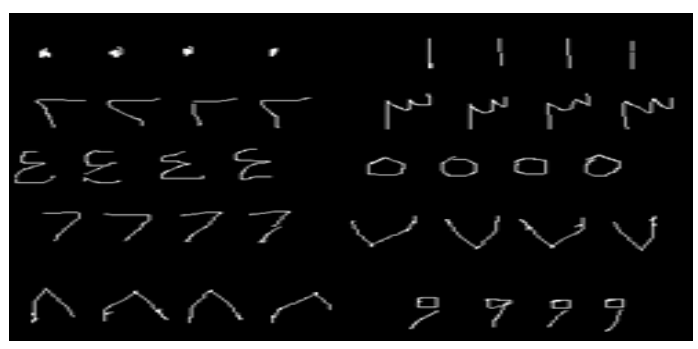

Figure 4. Example of some isolated handwritten Eastern Arabic numerals

We have chosen the following data:

1) Each original numeral image has a size equal to $30 \times 30$ pixels.

2) The size of the virtual retina equal to $5 \times 5,10 \times 10,15 \times 15$ and $20 \times 20$ pixels.

3) Each original numeral image is resized to $60 \times 60$ pixels.

4) Each numeral is transformed to a vector.

The standard deviation of the GRBF kernel function is equal to 0.1 .

Now, we group the values of the recognition rate $\mathbf{T}_{\mathbf{g}}$ (given in \%) for each numeral and also those of the global raterecognition i.e. of all numerals (given in \%) which we have obtained in the following table:

Table 2. The obtained recognition rates $\mathrm{Tn}$ and $\mathrm{tg}$ by each method of extraction

\begin{tabular}{|c|c|c|c|c|}
\hline & $T_{n(R C)}$ & $T_{n(R C)}$ & $T_{n(R C)}$ & $T_{n(R C)}$ \\
\hline Numerals & $5 \times 5$ & $10 \times 10$ & $15 \times 15$ & $20 \times 20$ \\
\hline . & 83 & 87 & 89 & 90 \\
\hline 1 & 70 & 79 & 92 & 97 \\
\hline r & 67 & 80 & 81 & 89 \\
\hline$r$ & 66 & 67 & 68 & 70 \\
\hline$\varepsilon$ & 74 & 75 & 77 & 80 \\
\hline 。 & 77 & 78 & 81 & 93 \\
\hline 9 & 60 & 70 & 75 & 80 \\
\hline$v$ & 80 & 81 & 84 & 88 \\
\hline$\wedge$ & 79 & 83 & 84 & 90 \\
\hline 9 & 60 & 62 & 65 & 69 \\
\hline$T_{g}$ & 71,6 & 76,2 & 79,6 & 84,6 \\
\hline
\end{tabular}

Isolated Handwritten Eastern Arabic Numerals Recognition Using Support... (B. El Kessab) 
Table 3. The obtained recognition rates for all numerals

\begin{tabular}{|c|c|c|c|c|c|c|c|c|c|c|}
\hline Numerals & $\cdot$ & 1 & $r$ & $r$ & $\varepsilon$ & 0 & 7 & $\mathrm{~V}$ & $\Lambda$ & 9 \\
\hline$\cdot$ & 90.00 & 3.00 & 0.00 & 0.01 & 0.40 & 0.27 & 0.22 & 0.17 & 0.36 & 0.21 \\
\hline 1 & 7.19 & 97.00 & 0.23 & 0.64 & 0.02 & 0.48 & 0.44 & 0.18 & 0.33 & 2.59 \\
\hline r & 0.19 & 0.00 & 89.00 & 23.30 & 9.00 & 0.65 & 5.00 & 3.41 & 1.20 & 0.93 \\
\hline$r$ & 0.16 & 0.00 & 9.96 & 70.00 & 8.00 & 0.45 & 3.00 & 6.23 & 2.95 & 0.42 \\
\hline$\varepsilon$ & 0.22 & 0.00 & 0.00 & 1.70 & 80.00 & 0.85 & 3.30 & 0.28 & 0.59 & 0.83 \\
\hline • & 0.13 & 0.00 & 0.00 & 0.00 & 0.60 & 93.00 & 0.03 & 0.22 & 0.58 & 0.92 \\
\hline 7 & 0.29 & 0.00 & 0.81 & 3.00 & 0.40 & 0.43 & 80.00 & 0.00 & 1.78 & 23.45 \\
\hline$v$ & 0.15 & 0.00 & 0.00 & 0.64 & 0.00 & 0.74 & 0.00 & 88.00 & 0.84 & 0.79 \\
\hline$\wedge$ & 0.82 & 0.00 & 0.00 & 0.26 & 0.08 & 0.34 & 0.70 & 1.12 & 90.00 & 0.86 \\
\hline 9 & 0.85 & 0.00 & 0.00 & 0.45 & 1.50 & 2.79 & 7.31 & 0.39 & 1.37 & 69.00 \\
\hline
\end{tabular}

The graphical representation to recognition rate of each numeral $\mathbf{T}_{\mathbf{n}}$ is:

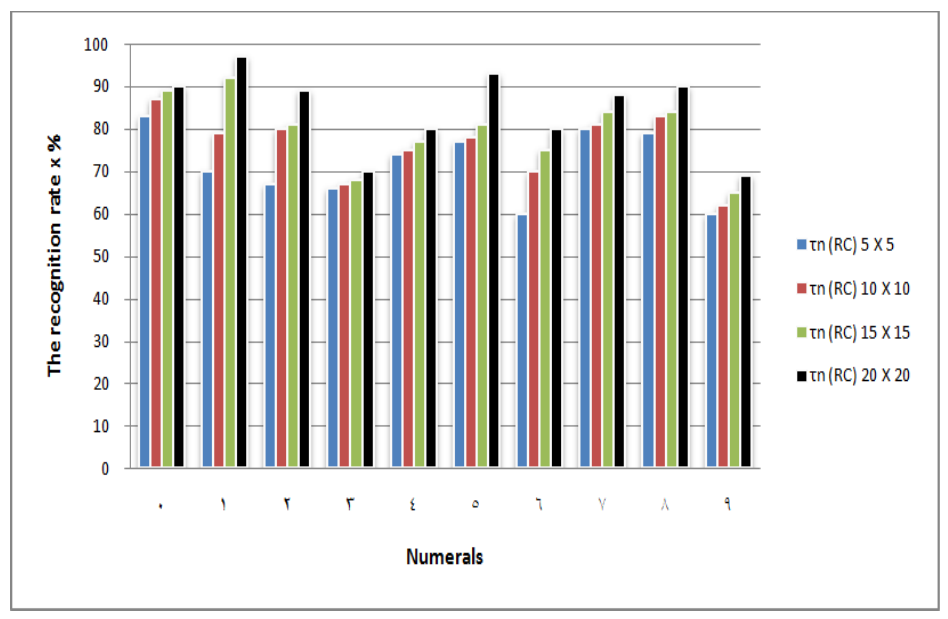

Figure 5. The graphical representation of recognition rate $T_{n}$ of each method of extraction

The graphical representation to recognition rate of all numerals $\mathbf{T}_{\mathbf{g}}$ is presented in the following figure:

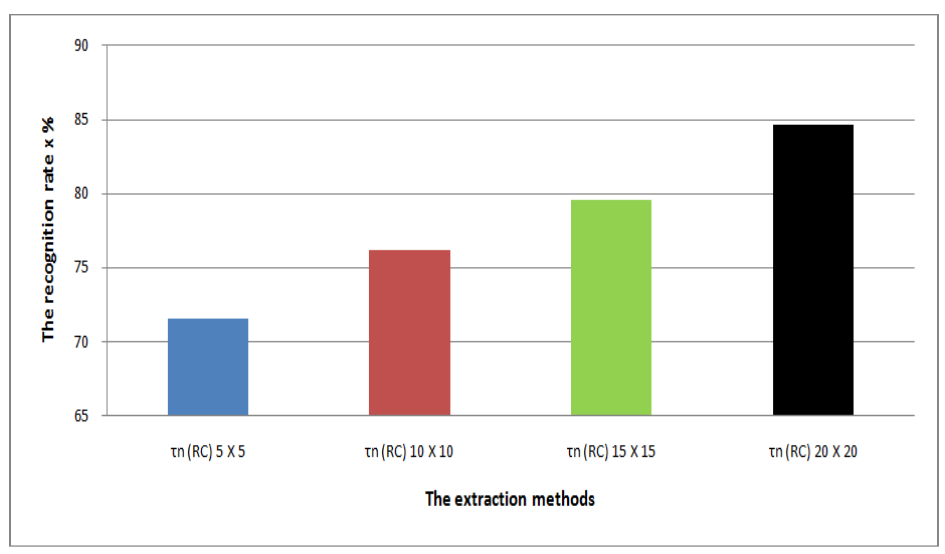

Figure 6. The graphical representation of global rate recognition $\mathrm{T}_{\mathrm{g}}$ of each method of extraction

\subsection{Analysis and Comment}

Taking into account all the results that we obtained, we really can to conclurethat:The most performant method is the retinal coding withvirtual retinasize equal $20 \times 20$ followed byretinal codingwith virtual retinasize equal $15 \times 15$ then the retinal coding withvirtual retinasize equal $10 \times 10$ then finaly the retinal coding withvirtual retinasize equal $5 \times 5$. 


\section{Conclusion}

In this paper, we have presented a comparison between the performances of several sizes of virtual retinawhich are the support vectors machines used for recognition of isolated Eastern Arabic handwritten numerals. In this sense we have verified that the recognition systems used in this approach which contains in the preprocessing phase the median filter, the thresholding and the centering and the support vectors machine in the recognition phasereally shows that the most powerful recognition system is that contains the retinal coding with virtual retinasize equal $20 \times 20$.

\section{Acknowledgements}

We are very grateful to our professors Mister Cherki Daoui and Mister BelaidBouikhalene for their continuous encouragements, their great cooperation, their pertinent advices, their appropriate guidance in the realization of this work. Many thanks again to them.

\section{References}

[1] Shahrul Nizam Yaakob1, Puteh Saad. Krawtchouk Moment Invariant And Gaussian ARTMAP Neural Network: A Combination Techniques For Image Classification. KUKUM Engineering Research Seminar. 2006.

[2] Hamid Reza Boveiri. On Pattern Classification Using Statistical Moments. International Journal of Signal Processing, Image Processing and Pattern Recognition. 2010; 3(4): 2010.

[3] Anass El affar Khalid Ferdous, Abdeljabbar Cherkaoui Hakim El fadil, Hassan Qjidaa. Krawtchouk Moment Feature Extraction for Neural Arabic Handwritten Words Recognition. IJCSNS International Journal of Computer Science and Network Security. 2009; 9(1).

[4] P Nagabhushan, SA Angadi, BS Anami. A fuzzy statistical approach of Kannada vowel recognition based on invariant moments. NCDAR. Mandy, India. 2003: 275-285.

[5] Ibrahim A El rube', Mohamed T El Sonni, Soha S Saleh. Printed Arabic sub-word recognition using moments. World Academy of Science, Engineering and Technology. 2010; 42.

[6] Srivastava DK, Bhambhu L. Data classification using Support Vector Machine. International Journal of Theoretical and Applied Information Technology. 2009; 12(1): 1-7.

[7] J Jindal, A Dhir R. Rani R. Diagonal features and SVM classifier for handwritten Gurumukhi character recognition. International Journal of Advanced Research in Computer Science and Software Engineering. 2012; 2(5): 505-508.

[8] DC Shubhangi, PS Hiremath. Handwritten English character and digit recognition using multiclass SVM classifier and using structural micro features. International Journal of Recent Trends in Engineering. 2009; 2(2).

[9] G Sinha, J kumar. Arabic numeral Recognition Using SVM Classifier. International Journal of Emerging Research in Management \& Technology. 2013; 2(5).

[10] A Hennig, N Sherkat. Exploiting zoning based on approximating splines in cursive script recognition. Pattern Recognition. 2002; 35(2): 445-454.

[11] B El kessab, C Daoui, B Bouikhalene, M Fakir, K Moro. Extraction Method of Handwritten Digit Recognition Tested on the MNIST Database. International Journal of Advanced Science and Technology. 2013; 50.

[12] RS Hegadi. Recognition of Printed Kannada Numerals based on Zoning Method. International Journal of Computer Applications (0975-8878) on National Conference of Advanced Computing and Communications - NCACC. 2012.

[13] A Hennig, N Sherkat. Exploiting zoning based on approximating splines in cursive script recognition. Pattern Recognition. 2002; 35(2): 445- 454.

[14] D Impedovo, G Pirlo. Zoning methods for handwritten character recognition: A survey. Pattern Recognition. 2014; 47(3): 969-981.

[15] M Kamel, A Campilho. An Iris Recognition Method Based On Zigzag Collarette Area and Asymmetrical Support Vector Machines. ICIAR. 2007: 854-865.

[16] M Padmaa, Y Venkataramani. ZIG-ZAG PVD - A Nontraditional Approach. International Journal of Computer Applications. 2010; 5(7).

[17] K Roy, P Bhattacharya. An Iris Recognition Method Based On Zigzag Collarette Area and Asymmetrical Support Vector Machines. IEEE. 2006: 861-865. 